\title{
Putting guidelines into practice: has the era of perioperative chemotherapy arrived?
}

\author{
Chloe L. Georgiou ${ }^{1}$, Carmel Pezaro ${ }^{1,2}$, Shomik Sengupta ${ }^{2,3}$ \\ ${ }^{1}$ Department of Medical Oncology, Eastern Health, Box Hill, Victoria, Australia; ${ }^{2}$ Eastern Health Clinical School, Monash University, Box Hill, \\ Victoria, Australia; ${ }^{3}$ Department of Urology, Eastern Health, Box Hill, Victoria, Australia \\ Correspondence to: Shomik Sengupta. Eastern Health Clinical School, Level 2, 5 Arnold Street, Box Hill, Victoria 3128, Australia. \\ Email: shomik.sengupta@monash.edu. \\ Provenance: This is a Guest Editorial commissioned by Section Editor Xiao Li (Department of Urology, Jiangsu Cancer Hospital \& Jiangsu Institute \\ of Cancer Research \& Nanjing Medical University Affiliated Cancer Hospital, Nanjing, China). \\ Comment on: Booth CM, Karim S, Brennan K, et al. Perioperative chemotherapy for bladder cancer in the general population: Are practice patterns \\ finally changing? Urol Oncol 2018;36:89.e13-89.e20.
}

Submitted Mar 23, 2018. Accepted for publication Mar 29, 2018.

doi: $10.21037 /$ tau.2018.04.02

View this article at: http://dx.doi.org/10.21037/tau.2018.04.02

In the current landscape of bladder cancer management, marked survival differences exist between non-muscle invasive, muscle invasive and metastatic disease (5-year survival $95 \%, 50 \%$ and $6 \%$, respectively) (1). The rationale for a multi-modal approach to muscle-invasive bladder cancer (MIBC) is to maximize curative treatment outcomes and prevent progression to metastatic disease. For surgical management, radical cystectomy is the definitive treatment option. Peri-operative chemotherapy offers additional benefit in disease free interval and overall survival (2), however utilization rates continue to be relatively low worldwide. The study by Booth $e t a l$. is a large populationbased analysis of time trends in use of peri-operative chemotherapy for MIBC in Ontario, Canada (3).

The extensive variation in practice patterns may be partly due to a lack of consensus regarding the optimal sequence of treatment. Consensus international guidelines (4-6) recommend neo-adjuvant chemotherapy for MIBC based on level 1 evidence from meta-analyses, which demonstrate an improvement in overall survival by about $5 \%$ (7-9). Although similar effects are also evident in some trials of adjuvant chemotherapy, smaller sample sizes, variability in study design and inconsistent results make these data less robust (10). There are some data suggesting progressionfree survival benefit in adjuvant $v$ s. delayed post-operative chemotherapy, but no difference in overall survival (11). To date, there has been no direct comparison between neoadjuvant and adjuvant chemotherapy.
From a practical perspective, neo-adjuvant chemotherapy may be more easily delivered in a prescribed, planned schedule in the lead up to radical cystectomy. Additional benefits include downstaging of disease with increased chance of $\mathrm{T} 0$ stage at resection, potentially leading to greater ease of surgery. Improved prognostication based on histology at time of cystectomy has also been demonstrated (12). This approach also avoids delays and failure to undergo adjuvant treatment, which can occur due to slow postoperative recovery and return to baseline performance status, as well as unexpected post-operative complications.

Conversely, there may be clinical rationale to progress immediately to resection and subsequently consider adjuvant treatment. This approach may mitigate against concerns regarding the delay to cystectomy, which has repeatedly been found to decrease overall survival (13). Additionally, radical cystectomy can potentially demonstrate histopathological factors including nodal status and extravesical extension, which are associated with higher risk of recurrent or metastatic disease, thereby justifying the risks and morbidity of chemotherapy. On the other hand, accurate pathological staging at the time of resection may also identify patients with favorable features who may derive less benefit and therefore avoid adjuvant chemotherapy. The decision to proceed straight to definitive surgical management also captures patients who may have otherwise had delay or been unfit for surgery due to chemotherapy toxicities. 
In addition to the controversy regarding the timing of chemotherapy in relation to cystectomy, other factors that may limit its usage include patient fitness and preferences as well as clinician preferences. In an effort to understand these factors further, Booth et al. conducted a retrospective analysis of 5,582 cystectomy patients treated in Ontario over a 20-year period from 1994-2013 (3). Such a substantial collection of data allows for comprehensive analysis of practice patterns in the field of MIBC treatment. Primary outcomes evaluated the rate of perioperative chemotherapy utilization, as well as referral to medical oncology. This article builds on previous work establishing trends from 1994-2008 (14) and compares contemporary results from 2009-2013.

In this study, the use of pathology reports from time of cystectomy added a level of data integrity to the identification of cases. These were then correlated with billing records for medical oncologists and chemotherapy codes within a 16 -week perioperative time frame. This process likely captured the majority of cases who received neoadjuvant or adjuvant chemotherapy, and in itself was no mean feat. However, this may have unintentionally excluded cases in which an informal oncology opinion was sought, including in multidisciplinary meetings (MDMs).

In terms of their primary endpoint, Booth et al. demonstrated a significant increase in perioperative chemotherapy utilization, with an OR of 5.17 for patients undergoing radical cystectomy in 2009-2013 compared to the earlier timeframe. A significant trend was seen towards increased peri-operative referral to medical oncology in the contemporary era (from 12\% in 1994-1998 to $32 \%$ in 2009-2013). For patients seen pre-operatively, there was also a substantial increase in the use of neoadjuvant chemotherapy (from 5\% in 1994-1998 to 19\% in 2009-2013).

Post-operatively, node positivity at cystectomy was correlated with a higher referral rate to medical oncology and a greater than three-fold increase in the prescription of adjuvant chemotherapy. This is a change in practice that has been reported by other groups (15), and the authors acknowledged the potential influence of previous papers and release of consensus guidelines. Whilst this demonstrates an encouraging trend, the overall rate remains low and further highlights the need to identify factors preventing appropriate uptake of perioperative chemotherapy.

In this paper, confounders such as age, geographic location, socioeconomic status, medical comorbidities, and volume of surgical experience per clinician have been taken into account. The authors acknowledged potential bias due to inability to evaluate lack of treatment based on patient factors, including preference for surgical treatment alone, or social factors including financial difficulty or absence from work. Data on performance status and creatinine clearance were also not available, two factors which would identify patients in whom cisplatin containing regimes or any perioperative chemotherapy may have been deemed inappropriate.

Within the limitations of a retrospective study, Booth et al. identify multiple factors that may contribute to the disparity in practice and referral patterns. An assessment of referral rate adjusted for volume of cases per surgeon and hospital district intriguingly implies significant practitionerbased variability. This may provide a valuable opportunity to target further interventions towards improving referral rates at low-outlying centers. Given this established dataset, a potential continuation of this project could involve an intervention surrounding education patterns and streamlined referral processes to optimize patient referrals from surgeon to medical oncologist.

When reporting on their secondary objective, Booth et al. discuss the utilization of MDM discussion to identify patients who would benefit from perioperative treatment. Participation in the MDM process has been found to increase rates of referral and perioperative chemotherapy uptake, and development of a robust MDM program in health networks may be one systemic approach which increases capture of appropriate cases in a timely fashion (16). The identification of a subgroup of patients who may have received further treatment if referred to medical oncologists is perhaps the most novel aspect of this paper. This highlights the need for a system to identify cases at initial diagnosis and ensure timely referral and treatment. Whilst not all cases treated with cystectomy may be appropriate for perioperative chemotherapy, sophisticated analysis has found that $51 \%$ of cases in this series may have obtained benefit, indicating that many may have missed out.

The current treatment landscape in non-metastatic bladder cancer is changing, with improved understanding of the biology on a cellular and genetic level (17). Through genomic sequencing, specific genes have been identified which may assist in the identification of patients most likely to benefit from neo-adjuvant chemotherapy (18). Clinical trials are also currently underway to assess the use of immunotherapy agents as adjuncts to surgical management (clinicaltrials. gov identifier NCT02450331, NCT01435356) (19), and results of these studies are eagerly awaited.

The data presented by Booth et al. comprehensively narrates the story of a population wide shift in treatment patterns and approach to MIBC, and mirrors observations 
of similar practice changes in other studies. Whilst there are many exciting developments in the field, we have the current ability to improve the application of best practice treatment in a multidisciplinary, patient centered approach. It is crucial to ensure that patients who may benefit from perioperative chemotherapy are provided with the opportunity to discuss available options and make informed treatment decisions.

\section{Acknowledgements}

None.

\section{Footnote}

Conflicts of Interest: The authors have no conflicts of interest to declare.

\section{References}

1. Abraham J, Gulley JL, Allegra CJ. The Bethesda Handbook of Clinical Oncology. 4th edition. Philadelphia: Lippincott Williams \& Wilkins, 2014.

2. Winquist E, Kirchner TS, Segal R, et al. Neoadjuvant chemotherapy for transitional cell carcinoma of the bladder: a systematic review and meta-analysis. J Urol 2004;171:561-9.

3. Booth CM, Karim S, Brennan K, et al. Perioperative chemotherapy for bladder cancer in the general population: Are practice patterns finally changing? Urol Oncol 2018;36:89.e13-89.e20.

4. Chang SS, Bochner BH, Chou R, et al. Treatment of NonMetastatic Muscle-Invasive Bladder Cancer: AUA/ASCO/ ASTRO/SUO Guideline. J Urol 2017;198:552-9.

5. Witjes JA, Compérat E, Cowan NC, et al. EAU Guidelines on Muscle-invasive and Metastatic Bladder Cancer: Summary of the 2013 Guidelines. Eur Urol 2014;65:778-92.

6. Bladder cancer: diagnosis and management of bladder cancer: (C) NICE (2015) Bladder cancer: diagnosis and management of bladder cancer. BJU Int 2017;120:755-65.

7. Advanced Bladder Cancer Meta-analysis Collaboration. Neoadjuvant chemotherapy in invasive bladder cancer: a systematic review and meta-analysis. Lancet 2003;361:1927-34.

8. Leow JJ, Martin-Doyle W, Rajagopal PS, et al. Adjuvant Chemotherapy for Invasive Bladder Cancer: A 2013 Updated Systematic Review and Meta-Analysis of
Randomized Trials. Eur Urol 2014;66:42-54.

9. Yin M, Joshi M, Meijer RP, et al. Neoadjuvant Chemotherapy for Muscle-Invasive Bladder Cancer: A Systematic Review and Two-Step Meta-Analysis. Oncologist 2016;21:708-15.

10. Sternberg CN, Sylvester R. Thoughts on a Systematic Review and Meta-analysis of Adjuvant Chemotherapy in Muscle-invasive Bladder Cancer. Eur Urol 2014;66:55-6.

11. Sternberg CN, Skoneczna I, Kerst JM, et al. Immediate versus deferred chemotherapy after radical cystectomy in patients with pT3-pT4 or N+M0 urothelial carcinoma of the bladder (EORTC 30994): an intergroup, open-label, randomised phase 3 trial. Lancet Oncol 2015;16:76-86.

12. Petrelli F, Coinu A, Cabiddu M, et al. Correlation of pathologic complete response with survival after neoadjuvant chemotherapy in bladder cancer treated with cystectomy: a meta-analysis. Eur Urol 2014;65:350-7.

13. Gore JL, Lai J, Setodji CM, et al. Mortality increases when radical cystectomy is delayed more than 12 weeks: results from a Surveillance, Epidemiology, and End ResultsMedicare analysis. Cancer 2009;115:988-96.

14. Booth CM, Siemens DR, Li G, et al. Perioperative chemotherapy for muscle-invasive bladder cancer: A population-based outcomes study. Cancer 2014;120:1630-8.

15. Liew MS, Azad A, Tafreshi A, et al. USANZ: Timetrends in use and impact on outcomes of perioperative chemotherapy in patients treated with radical cystectomy for urothelial bladder cancer. BJU Int 2013;112 Suppl 2:74-82.

16. Yau WH, Devitt B, Aung SY, et al. Clinical need for standardised multidisciplinary meeting assessment processes. Intern Med J 2018;48:230-1.

17. Pezaro C, Liew MS, Davis ID. Urothelial cancers: Using biology to improve outcomes. Expert Rev Anticancer Ther 2012;12:87-98.

18. Plimack ER, Dunbrack RL, Brennan TA, et al. Defects in DNA Repair Genes Predict Response to Neoadjuvant Cisplatin-based Chemotherapy in Muscle-invasive Bladder Cancer. Eur Urol 2015;68:959-67.

19. Singh P, Black P. Emerging role of checkpoint inhibition in localized bladder cancer. Urol Oncol 2016;34:548-55.

Cite this article as: Georgiou CL, Pezaro C, Sengupta S. Putting guidelines into practice: has the era of perioperative chemotherapy arrived? Transl Androl Urol 2018; 7(Suppl 2):S255S257. doi: 10.21037/tau.2018.04.02 